\title{
Corneal Endothelial Cell Loss after Phacoemulsification in Eyes with a Prior Acute Angle-closure Attack
}

\author{
Hosuck Yeom ${ }^{1}$, Eun Hee Hong ${ }^{1}$, Yong Un Shin ${ }^{1,2}$, Min Ho Kang ${ }^{1,2}$, Hee Yoon Cho ${ }^{1,2}$, Mincheol Seong ${ }^{1,2}$ \\ ${ }^{1}$ Department of Ophthalmology, Hanyang University Guri Hospital, Guri, Korea \\ ${ }^{2}$ Department of Ophthalmology, Hanyang University College of Medicine, Seoul, Korea
}

\begin{abstract}
Purpose: To evaluate endothelial damage after cataract surgery in eyes affected by an angle-closure attack (ACA) and compare it to that in the unaffected fellow eyes (FEs) of patients with ACA and normal eyes (NES).

Methods: The medical data of eyes affected by ACA, FEs (with no history of acute glaucoma attack), and NEs of patients who underwent cataract surgery with simultaneous intraocular lens implantation were retrospectively reviewed. Endothelial cell density (ECD) and central corneal thickness (CCT) measured before surgery and at 1 week, 1 month, and 3 months after surgery were analyzed, and the percentages of loss in ECD and increase in CCT of the three groups were compared.

Results: The study enrolled 140 eyes from 100 patients (50 eyes in the ACA group, 40 eyes in the FE group, and 50 eyes in the NE group). The mean ECD was significantly lower in the ACA group than in the other groups $(p<0.001)$. However, the percentage of ECD reduction was not significantly greater in the ACA group than in the other groups $(p>0.05)$. None of the eyes developed corneal edema at 3 months postoperatively. Moreover, the CCTs of the three groups were similar throughout the follow-up period $(p>0.05)$.

Conclusions: Phacoemulsification was not associated with greater endothelial cell loss in the ACA group than in the NE and FE groups. This finding shows that ACA history may not contribute to the exacerbation of corneal endothelial damage in cataract surgery.
\end{abstract}

Key Words: Angle closure, Corneal endothelial cell loss, Corneal pachymetry, Glaucoma, Phacoemulsification

Cataract surgery is the most frequently performed surgical procedure in the field of ophthalmology. Given the risk of corneal endothelial injury, the cornea should be thoroughly evaluated before cataract surgery. However, safety standards delineating the preoperative endothelial cell density (ECD) necessary to maintain a clear cornea after cata-

Received: March 27, 2020 Final revision: August 4, 2020

Accepted: August 6, 2020

Corresponding Author: Mincheol Seong, MD, PhD. Department of Ophthalmology, Hanyang University Guri Hospital, \#153 Gyeongchun-ro, Guri 11923, Korea. Tel: 82-31-560-2354, Fax: 82-31-564-9479, E-mail: goddns76@hanmail.net ract surgery are lacking [1]

In patients with primary angle-closure, an acute glaucoma attack can damage the corneal endothelium because of elevated intraocular pressure, as seen in mechanical trauma. Therefore, the cornea weakens and becomes more vulnerable to damage during intraocular surgery [2-4]. Previous publications showed decreased ECD in eyes with acute attacks [5-8]. Low ECD in patients with prior angle-closure attack (ACA) makes it challenging for surgeons to proceed with surgery [5-8]. There are a few reports on corneal endothelial damage caused by cataract surgery in eyes with significantly low ECD [1,9]. However, no study, to our 
knowledge, has documented corneal endothelial damage after cataract surgery in patients with prior ACA.

This study was designed to evaluate the risk of endothelial decompensation and the degree of corneal endothelial damage after cataract surgery in eyes with previous endothelial damage from an acute glaucoma attack. We hypothesized that both eyes from the same person mostly have similar conditions, including similar axial lengths (AXLs), anterior chamber depths (ACDs), iris and cornea contours, and lens thicknesses. To this end, we compared eyes with a history of acute ACA with two control groups: (1) the fellow eyes (FEs) without a history of glaucoma attacks of patients with angle-closure glaucoma (ACG) and (2) normal eyes (NEs) of patients with no previous ocular history. Our final analysis sought to determine the risk associated with only ACA.

The purpose of the current study was to evaluate endothelial damage after cataract surgery in eyes affected by ACA and compare this damage to that in the FEs and NEs.

\section{Materials and Methods}

The medical records of patients who underwent cataract phacoemulsification with simultaneous intraocular lens (IOL) implantation by one surgeon (MS) at Hanyang University Guri Hospital between January 2017 and March 2018 were reviewed. Informed consent was waived due to the retrospective nature of the study. We identified patients with previous ACA who underwent laser iridotomy to prevent recurrence (ACA group) and those with no history of ophthalmic disease (NE group). Patients who had experienced more than one ACA were excluded. If the FEs of patients with ACG who underwent cataract phacoemulsification had no history of ACA and underwent preventative laser iridotomy, they were included in the FE group. Approval for this study was obtained from the institutional review board (2013-06-031). All experiments were performed following the relevant guidelines and regulations.

\section{Surgical technique}

Cataract surgery was performed under topical anesthesia. A 2.8-mm clear corneal incision was made temporally. After the injection of sodium hyaluronate 1.4\% (Healon GV; Johnson \& Johnson Surgical Vision, Santa Ana, CA,
USA) into the anterior chamber to stabilize the chamber and protect endothelial cells, a continuous curvilinear capsulorrhexis was created with a bent 26-gauge needle. After hydrodissection, phacoemulsification of the nucleus and aspiration of the cortex were performed. The lens capsule was inflated with sodium hyaluronate 1.4\% (Healon GV), and a foldable acrylic IOL was placed in the capsular bag. The wound was closed with a 10-0 Ethilon simple suture. After surgery, moxifloxacin 0.5\% (Vigamox; Alcon Laboratories, Fort Worth, TX, USA) and prednisolone acetate 1\% (Pred Forte; Allergan, Irvine, CA, USA) were instilled four times daily for 3 weeks by all the patients.

\section{Main outcome measures}

All the eyes underwent preoperative ( 2 weeks before surgery) and postoperative (1 week, 1 month, and 3 months after surgery) evaluations. AXL and ACD were examined using noncontact partial coherence laser interferometry (Zeiss IOLMaster; Carl Zeiss AG, Oberkochen, Germany), while central corneal thickness (CCT) was examined using topical ultrasound pachymetry (Tomey SP-3000; Tomey, Erlangen, Germany). ECD was examined and photographed by experienced examiners using a noncontact specular microscope (SP 2000P; Topcon Corporation, Tokyo, Japan) $[10,11]$. The highest-quality video image was transmitted to an image-analysis computer, and the endothelial cells were counted manually. Any patient without a clear image of the endothelial cells was excluded. The intraoperative parameters were ultrasound time (UST; the total duration ultrasound energy was applied) and cumulative dissipated energy (CDE; the mean percentage of ultrasound power spent during the UST). Both UST and CDE values were automatically calculated by the phacoemulsification device and displayed on the monitor. Endothelial cell loss was evaluated using two newly defined parameters: ECD decrease ratio, calculated as (preoperative ECD - postoperative ECD) / preoperative ECD, and CCT increase ratio, calculated as (postoperative CCT - preoperative CCT) / preoperative CCT.

\section{Statistical analysis}

One-way analysis of variance was used to compare AXL, ACD, UST, CDE, ECD, and CCT among the three groups. Repeated-measures one-way analysis of variance 
was used to compare preoperative and postoperative (1 week, 1 month, and 3 months) ECD and CCT measurements among the three groups. If significant differences were detected among the three groups, Tukey's post hoc analysis was used to compare each pair of groups. Spear-

Table 1. Subject characteristics in the ACA group

\begin{tabular}{lc}
\hline Characteristics & Value \\
\hline Age (yr) & $67.6 \pm 9.5(45-84)$ \\
Sex (male : female) & $15: 35$ \\
Systemic disease & 19 \\
$\quad$ DM & 23 \\
HTN & $49.1 \pm 11.9(25-76)$ \\
Peak IOP (mmHg) & $35.7 \pm 31.0(6-140)$ \\
Mean duration of high IOP (hr) & $22.38 \pm 0.84$ \\
Axial length (mm) & $2.31 \pm 0.18$ \\
$\begin{array}{l}\text { Anterior chamber depth (mm) } \\
\text { Preoperative endothelial cell density } \\
\left.\quad \text { cells/mm }{ }^{2}\right)\end{array}$ & $1,818 \pm 490$ \\
$\begin{array}{l}\text { Preoperative central corneal thickness } \\
\text { ( } \mu \text { m) }\end{array}$ & $546.2 \pm 43.1$ \\
$\begin{array}{l}\text { Interval between ACA and } \\
\text { phacoemulsification (mon) }\end{array}$ & $2.17 \pm 1.71(1-8)$ \\
Presence of peripheral iridotomy & $50(100)$ \\
\hline
\end{tabular}

Values are indicated as mean \pm standard deviation (range), number, mean \pm standard deviation, or number (\%).

$\mathrm{ACA}=$ angle-closure attack; DM = diabetes mellitus; HTN = hypertension; IOP = intraocular pressure. man correlation analysis was used to detect significant differences between AXL and the ECD decrease ratio or $\mathrm{ACD}$ and the ECD decrease ratio. A $p$-value of $<0.05$ was considered statistically significant. The values are presented as means \pm standard deviation.

\section{Results}

The ACA group comprised 50 eyes from 50 patients, of whom 40 had undergone cataract surgery in the FEs, with 40 eyes included in the FE group. The NE group comprised 50 eyes from 50 patients. Table 1 shows the characteristics of the subject in the ACA group. In this group, the mean peak intraocular pressure (IOP) was $49.1 \pm 11.9$ $\mathrm{mmHg}$, and high IOP persisted for an average of $35.7 \pm$ 31.0 hours. Cataract surgery was performed for an average of $2.17 \pm 1.71$ months after the acute ACA. In all eyes of the ACA group, laser peripheral iridotomy was performed before the surgery.

Table 2 shows preoperative and intraoperative parameters in the three groups. The mean AXL was significantly lower in the ACA group than that in the NE group ( $p=$ $0.005)$. The mean ACD was significantly deeper in the NE group than in the ACA and FE groups $(p<0.001$ and $p=$ 0.002 , respectively). The mean preoperative ECDs were $1,818 \pm 490,2,633 \pm 395$, and 2,675 \pm 348 cells $/ \mathrm{mm}^{2}$ in the ACA, FE, and NE groups, respectively; the differences between the ACA and other groups were statistically significant ( $p<0.001$ for both groups). The mean preoperative

Table 2. Preoperative and intraoperative parameters in the ACA, FE, and NE groups

\begin{tabular}{lcccc}
\hline Group & ACA $(\mathrm{n}=50)$ & FE $(\mathrm{n}=40)$ & NE $(\mathrm{n}=50)$ & $p$-value \\
\hline AXL $(\mathrm{mm})$ & $22.38 \pm 0.84$ & $22.56 \pm 0.85$ & $23.44 \pm 1.39$ & $0.003^{*} / 0.919^{\dagger} / 0.005^{\ddagger} / 0.103^{\S}$ \\
ACD $(\mathrm{mm})$ & $2.31 \pm 0.18$ & $2.49 \pm 0.22$ & $3.02 \pm 0.52$ & $<0.001^{*} / 0.572^{\dagger} /<0.001^{\ddagger} / 0.002^{\S}$ \\
Preoperative ECD $\left(\right.$ cells $\left./ \mathrm{mm}^{2}\right)$ & $1,818 \pm 490$ & $2,633 \pm 395$ & $2,675 \pm 348$ & $<0.001^{*} /<0.001^{\dagger} /<0.001^{\ddagger} / 0.869^{\S}$ \\
Preoperative CCT $(\mu \mathrm{m})$ & $546.2 \pm 43.1$ & $544.0 \pm 43.2$ & $535.8 \pm 32.2$ & $0.514^{*}$ \\
UST $(\mathrm{sec})$ & $16.86 \pm 6.49$ & $18.22 \pm 10.94$ & $18.10 \pm 10.38$ & $0.855^{*}$ \\
CDE & $4.93 \pm 2.13$ & $4.96 \pm 2.61$ & $4.74 \pm 2.81$ & $0.942^{*}$ \\
\hline
\end{tabular}

Values are indicated as mean \pm standard deviation.

$\mathrm{ACA}=$ angle-closure attack; $\mathrm{FE}=$ fellow eye; $\mathrm{NE}=$ normal eye; $\mathrm{AXL}=$ axial length; $\mathrm{ACD}=$ anterior chamber depth; $\mathrm{ECD}=$ endothelial cell density; $\mathrm{CCT}=$ central corneal thickness; UST = ultrasound time; $\mathrm{CDE}=$ cumulative dissipated energy.

*One-way analysis of variance; Tukey's post hoc analysis ( ${ }^{\dagger}$ comparison between ACA and FE; ${ }^{*}$ comparison between ACA and NE; ${ }^{\S}$ comparison between FE and NE). 
Table 3. Endothelial cell density and central corneal thickness before and after cataract surgery in the ACA, FE, and NE groups

\begin{tabular}{llcccc}
\hline Group & & ACA $(\mathrm{n}=50)$ & FE $(\mathrm{n}=40)$ & NE $(\mathrm{n}=50)$ & $p$-value \\
\hline ECD & Before surgery & $1,818 \pm 490$ & $2,633 \pm 395$ & $2,675 \pm 348$ & $<0.001^{*} /<0.001^{\dagger} /<0.001^{\ddagger} / 0.869^{\S}$ \\
& 1 wk & $1,395 \pm 569$ & $2,006 \pm 458$ & $2,117 \pm 435$ & $<0.001^{*}$ \\
& 1 mon & $1,525 \pm 532$ & $2,249 \pm 496$ & $2,308 \pm 442$ & $<0.001^{*}$ \\
& 3 mon & $1,539 \pm 568$ & $2,225 \pm 408$ & $2,292 \pm 346$ & $<0.001^{*}$ \\
CCT & Before surgery & $546.2 \pm 43.1$ & $544.0 \pm 43.2$ & $535.8 \pm 32.2$ & $0.514^{*}$ \\
& 1 wk & $603.0 \pm 68.5$ & $591.1 \pm 52.8$ & $572.7 \pm 40.5$ & $0.070^{*}$ \\
& 1 mon & $564.6 \pm 39.2$ & $561.2 \pm 53.2$ & $555.2 \pm 40.4$ & $0.392^{*}$ \\
& 3 mon & $552.0 \pm 36.1$ & $549.7 \pm 48.9$ & $541.3 \pm 35.7$ & $0.521^{*}$ \\
\hline
\end{tabular}

Values are indicated as mean \pm standard deviation.

$\mathrm{ACA}=$ angle-closure attack; $\mathrm{FE}=$ fellow eye; $\mathrm{NE}=$ normal eye $\mathrm{ECD}=$ endothelial cell density; $\mathrm{CCT}=$ central corneal thickness.

"One-way analysis of variance; Repeated-measures one-way analysis of variance ('comparison between ACA and FE; tween ACA and NE; ${ }^{\S}$ comparison between FE and NE).
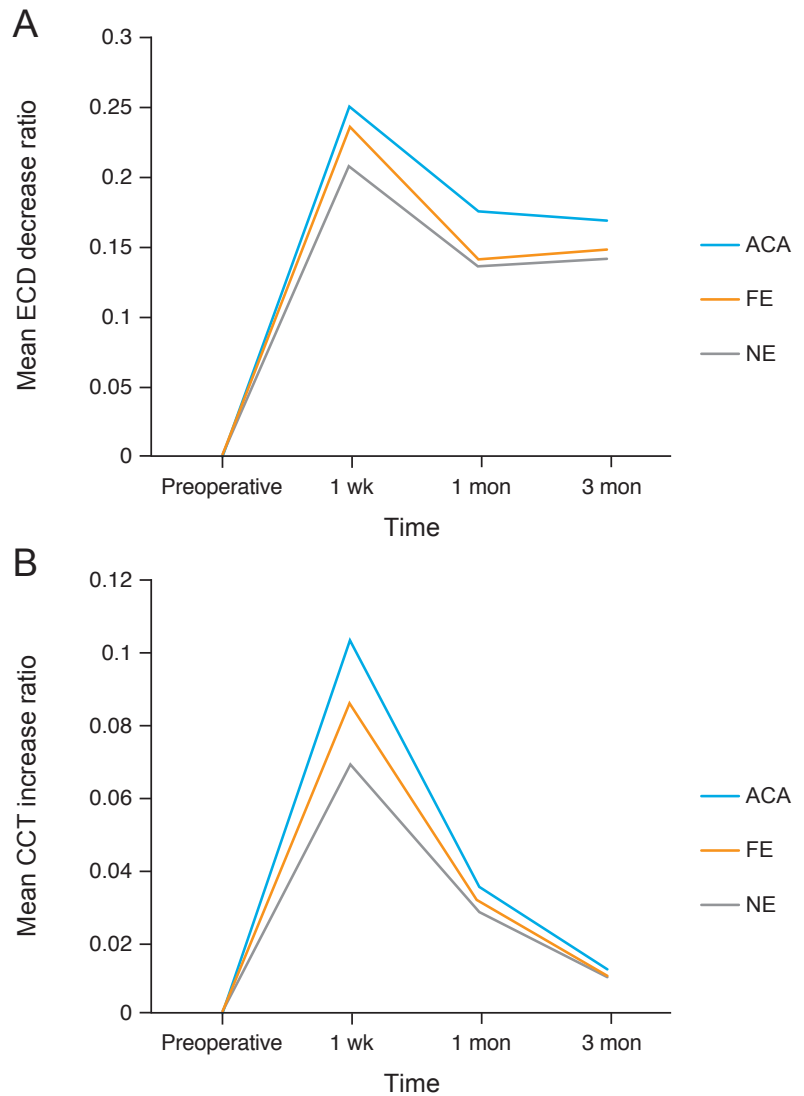

Fig. 1. Change in (A) endothelial cell density (ECD) and (B) central corneal thickness (CCT) after cataract surgery in the angle-closure attack (ACA), fellow eye (FE), and normal eye (NE) groups.
CCT values were $546.2 \pm 43.1,544.0 \pm 43.2$, and $535.8 \pm$ $32.2 \mu \mathrm{m}$ in the ACA, FE, and NE groups, respectively ( $p=$ $0.514)$. No statistically significant differences were observed in the UST and CDE among the three groups ( $p=$ 0.855 and 0.942 , respectively).

The mean ECD was significantly lower in the ACA group than in the other groups throughout the follow-up period (all $p<0.001$ ). In contrast, no statistically significant differences were observed in mean CCT among the three groups throughout the follow-up period ( $p=0.070$, 0.392 , and 0.521 at 1 week, 1 month, and 3 months after surgery, respectively) (Table 3). In the ACA group, the mean ECD 3 months postoperatively was 1,539 \pm 568 cells/ $\mathrm{mm}^{2}$, but two eyes had values of $>2,000$ cells $/ \mathrm{mm}^{2}(2,225$ and 2,292 cells $/ \mathrm{mm}^{2}$ ).

The mean ECD decrement ratios 3 months after surgery were $0.169 \pm 0.120,0.147 \pm 0.149$, and $0.140 \pm 0.105$ in the ACA, FE, and NE groups, respectively. Similarly, the mean CCT increase ratios 3 months after surgery were $0.012 \pm 0.039,0.010 \pm 0.017$, and $0.011 \pm 0.038$ in the ACA, FE, and NE groups, respectively. No statistically significant differences in ECD or CCT ratio changes were observed among the three groups throughout the follow-up period (all $p>0.05$ ) (Table 4 and Fig. 1A, 1B).

Among the three groups, the associations between AXL and the ECD decrease and CCT increase ratios were not statistically significant (Fig. 2A, 2B). However, negative linear relationships were observed between the ACD and 
Table 4. Change in endothelial cell density and central corneal thickness after cataract surgery in the ACA, FE, and NE groups

\begin{tabular}{llllll}
\hline Group & & ACA $(\mathrm{n}=50)$ & FE $(\mathrm{n}=40)$ & NE $(\mathrm{n}=50)$ & $p$-value \\
\hline ECD decrease ratio & $1 \mathrm{wk}$ & $0.250 \pm 0.150$ & $0.235 \pm 0.143$ & $0.207 \pm 0.144$ & $0.521^{*}$ \\
& $1 \mathrm{mon}$ & $0.174 \pm 0.098$ & $0.141 \pm 0.016$ & $0.136 \pm 0.140$ & $0.574^{*}$ \\
& $3 \mathrm{mon}$ & $0.169 \pm 0.120$ & $0.147 \pm 0.149$ & $0.140 \pm 0.105$ & $0.650^{*}$ \\
CCT increase ratio & $1 \mathrm{wk}$ & $0.103 \pm 0.077$ & $0.086 \pm 0.042$ & $0.069 \pm 0.043$ & $0.075^{*} / 0.854^{\dagger} / 0.342^{\ddagger} / 0.878^{\S}$ \\
& $1 \mathrm{mon}$ & $0.035 \pm 0.048$ & $0.031 \pm 0.031$ & $0.028 \pm 0.041$ & $0.803^{*}$ \\
\hline
\end{tabular}

Values are indicated as mean \pm standard deviation; ECD decrease ratio $=($ preoperative ECD - postoperative ECD) / preoperative ECD; $\mathrm{CCT}$ increase ratio $=($ postoperative $\mathrm{CCT}-$ preoperative $\mathrm{CCT}) /$ preoperative CCT .

$\mathrm{ACA}=$ angle-closure attack; $\mathrm{FE}=$ fellow eye; $\mathrm{NE}=$ normal eye; $\mathrm{ECD}=$ endothelial cell density; $\mathrm{CCT}=$ central corneal thickness.

*One-way analysis of variance; Repeated-measures one-way analysis of variance ('comparison between ACA and FE; ${ }^{*}$ comparison between ACA and NE; ${ }^{\S}$ comparison between FE and NE).
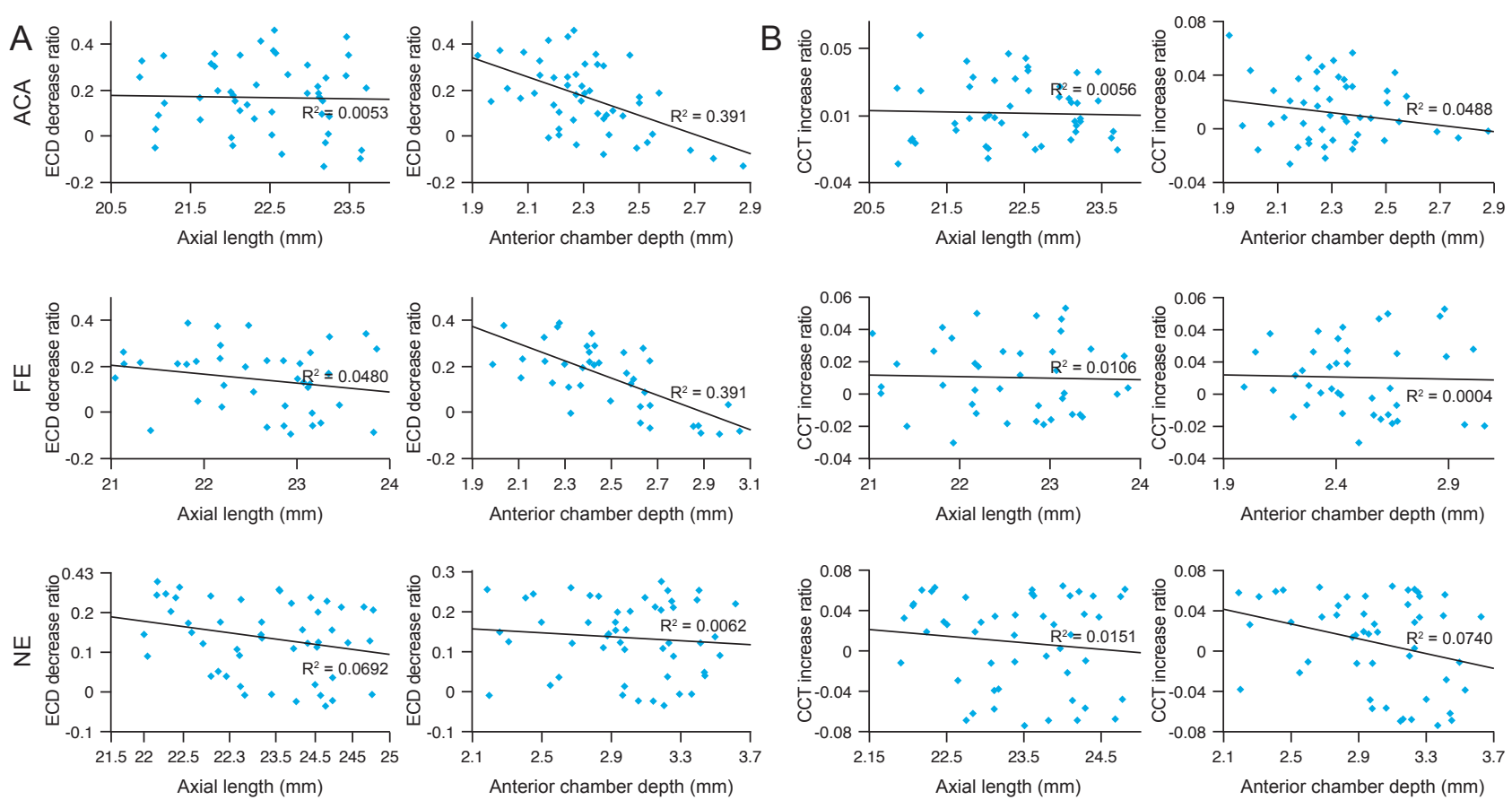

Fig. 2. Correlation coefficients between (A) the endothelial cell density (ECD) decrease ratio and axial length or anterior chamber depth and $(\mathrm{B})$ the central corneal thickness $(\mathrm{CCT})$ increase ratio and axial length or anterior chamber depth at 3 months after surgery. ACA = angle-closure attack; $\mathrm{FE}=$ fellow eye; $\mathrm{NE}=$ normal eye .

ECD decrement ratios in all the three groups $(p=0.004$, 0.015 , and 0.048 in the ACA, FE, and NE groups, respectively). Finally, none of the eyes from any group showed persistent corneal edema within 3 months after surgery, and none of the eyes showed severe bullous keratopathy.

\section{Discussion}

Our study found that the corneal endothelial cell loss after phacoemulsification in the ACA group was not different from that in the other groups. Previous damage from high IOP likely created a harsh corneal environment that 
may have worsened after intraocular surgery $[12,13]$. Before this study, we expected the ACA group to experience more damage than the NE group, because fewer endothelial cells occupied the same surface area in a low ECD endothelium. Our postoperative investigation showed that, while the ECD in the ACA group was low, in comparison to the preoperative values, the ECD decrease ratio was not significantly higher than those of the other two groups.

ECD remarkably decreased at 1 week after surgery and started to gradually recover over 1 month. The CCT peaked at 1 week after surgery, and it had recovered to close to the preoperative values at 3 months after surgery. Endothelial rearrangement was likely induced by endothelial concentric migration during the healing process. As a consequence of the recovered function, the corneal edema gradually decreased $[14,15]$.

Three months after surgery, the CCT in the ACA group did not differ significantly from those in the other groups. Despite the low ECD, the corneal endothelium in the ACA group maintained corneal homeostasis and showed compensated endothelial function. Therefore, in our study, CCT served as a better parameter than ECD for assessing overall endothelial function [11,14]. In the ACA group, which had low ECD, CCT did not significantly increase between the preoperative and postoperative periods.

Previous studies have found that CCT returns to values similar to the preoperative values 3 months after cataract surgery, regardless of the severity of endothelial cell loss [15-19]. These findings reflected our results. Furthermore, another study suggested that the duration of the healing process (i.e., restoring the preoperative CCT value) depends on the prevalence of endothelial diseases such as Fuchs' disease [20]. Nevertheless, in our study, no difference was observed between the recovery times of the ACA and NE groups.

The decrease in ECD during cataract surgery could have been influenced by other factors, including short AXL, shallow ACD, and cataract severity. To identify the effect of these factors, the ACA group was compared to the FE group. No significant differences in ACD and AXL were observed between these two groups. The FE group showed intermediate ECD decrease and $\mathrm{CCT}$ increase ratios between the ACA and NE groups. However, the difference was not statistically significant. Other factors may have influenced endothelial damage in the ACA and FE groups. These two groups experienced more endothelial damage than the NE group because of difficult surgical environments such as short AXL, shallow ACD, and small eyes, even if not previously damaged $[12,13]$. This suggests that a history of ACA affects postoperative prognosis due to general factors related to the difficulty of surgery rather than the low ECD status.

In our study, a significant negative correlation was observed between the ACD and ECD decrement ratios in all three groups. During surgery, ACD affected the instrument position and endothelial injury risk from phacoemulsification; the injury may be aggravated by a shallow ACD. Without considering other factors, ACD showed a strong negative correlation with ECD decrement ratios in the ACA and FE groups. Nevertheless, unlike in previous studies, AXL was not significantly correlated with endothelial cell change in this study $[12,13]$.

None of the patients in the study developed endothelial dysfunction after cataract surgery; therefore, we were unable to measure the association between ECD and bullous keratopathy. However, the risk of endothelial decompensation in eyes with ACG did not seem significant.

This study has several limitations. First, other putative factors that may have influenced ECD from cataract surgery (i.e., cataract severity and type) were not fully considered. We used the FE group as a control to reduce the error that could result from those factors. However, this was not enough to significantly reduce errors. Second, the short follow-up period may have resulted in our neglect of delayed-onset complications. Previous studies had assumed that a 3-month follow-up was enough to compare the ACA and normal groups. However, we cannot rule out the possibility that endothelial decompensation, such as bullous keratopathy, could have been observed with a longer follow-up. Third, we did not evaluate the endothelial cell morphology. Morphological criteria, rather than cell counts, may play a more formative role in endothelial function. Finally, we did not check the presence and severity of glaucomflecken during or after ACA. Further investigations are needed to determine if such factors affecting endothelial conditions should be checked before surgery.

In patients with $\mathrm{ACG}$, cataract progression aggravates glaucomatous conditions and increases the risk of ACA. However, low ECD in patients with ACG makes it challenging for surgeons to proceed with surgery.

The results of this study showed that cataract surgery in patients with ACA is not as risky for corneal endothelial 
decompensation as it is commonly expected. Cataract surgery may be a better option for preventing ACA before the choice of surgery becomes more difficult. Understanding the implications of short AXL and shallow ACD, with significant attention to the baseline ECD, will guarantee a safer surgical procedure.

\section{Conflict of Interest}

No potential conflict of interest relevant to this article was reported.

\section{References}

1. Yamazoe K, Yamaguchi T, Hotta K, et al. Outcomes of cataract surgery in eyes with a low corneal endothelial cell density. J Cataract Refract Surg 2011;37:2130-6.

2. Shah S, Chatterjee A, Mathai M, et al. Relationship between corneal thickness and measured intraocular pressure in a general ophthalmology clinic. Ophthalmology 1999;106:2154-60.

3. Copt RP, Thomas R, Mermoud A. Corneal thickness in ocular hypertension, primary open-angle glaucoma, and normal tension glaucoma. Arch Ophthalmol 1999;117:14-6.

4. Gagnon MM, Boisjoly HM, Brunette I, et al. Corneal endothelial cell density in glaucoma. Cornea 1997;16:314-8.

5. Olsen T. The endothelial cell damage in acute glaucoma. On the corneal thickness response to intraocular pressure. Acta Ophthalmol (Copenh) 1980;58:257-66.

6. Sihota R, Lakshmaiah NC, Titiyal JS, et al. Corneal endothelial status in the subtypes of primary angle closure glaucoma. Clin Exp Ophthalmol 2003;31:492-5.

7. Malaise-Stals J, Collignon-Brach J, Weekers JF. Corneal endothelial cell density in acute angle-closure glaucoma. Ophthalmologica 1984;189:104-9.

8. Ko YC, Liu CJ, Lau LI, et al. Factors related to corneal endothelial damage after phacoemulsification in eyes with occludable angles. J Cataract Refract Surg 2008;34:46-51.
9. Hayashi K, Yoshida M, Manabe S, Hirata A. Cataract surgery in eyes with low corneal endothelial cell density. $J$ Cataract Refract Surg 2011;37:1419-25.

10. Bovelle R, Kaufman SC, Thompson HW, Hamano H. Corneal thickness measurements with the Topcon SP-2000P specular microscope and an ultrasound pachymeter. Arch Ophthalmol 1999;117:868-70.

11. Modis L Jr, Langenbucher A, Seitz B. Corneal endothelial cell density and pachymetry measured by contact and noncontact specular microscopy. J Cataract Refract Surg 2002;28:1763-9.

12. Walkow T, Anders N, Klebe S. Endothelial cell loss after phacoemulsification: relation to preoperative and intraoperative parameters. J Cataract Refract Surg 2000;26:72732.

13. Hayashi K, Hayashi H, Nakao F, Hayashi F. Risk factors for corneal endothelial injury during phacoemulsification. J Cataract Refract Surg 1996;22:1079-84.

14. Ventura AC, Walti R, Bohnke M. Corneal thickness and endothelial density before and after cataract surgery. $\mathrm{Br} J$ Ophthalmol 2001;85:18-20.

15. Cheng H, Bates AK, Wood L, McPherson K. Positive correlation of corneal thickness and endothelial cell loss. Serial measurements after cataract surgery. Arch Ophthalmol 1988;106:920-2.

16. Kohlhaas M, Stahlhut O, Tholuck J, Richard G. Changes in corneal thickness and endothelial cell density after cataract extraction using phacoemulsification. Ophthalmologe 1997;94:515-8.

17. Amon M, Menapace R, Radax U, Papapanos P. Endothelial cell density and corneal pachometry after no-stitch, small-incision cataract surgery. Doc Ophthalmol 1992;81:301-7.

18. Olsen T. Corneal thickness and endothelial damage after intracapsular cataract extraction. Acta Ophthalmol (Copenh) 1980;58:424-33.

19. Olsen T, Eriksen JS. Corneal thickness and endothelial damage after intraocular lens implantation. Acta Ophthalmol (Copenh) 1980;58:773-86.

20. Saini JS, Mittal S. In vivo quantification of corneal endothelium function. Acta Ophthalmol Scand 1996;74:468-72. 